\title{
Temporal trend, clinicopathologic and sociodemographic characterization of age at diagnosis of breast cancer among US women diagnosed from 1990 to 2009
}

\author{
Gabriel Escarela*, Luis Carlos Pérez-Ruiz and Gabriel Núñez-Antonio
}

\begin{abstract}
This paper investigates the distribution of age at diagnosis of female breast cancer and its association with temporal trend, clinicopathologic and sociodemographic variables in the presence of two latent clusters that are directly unobservable. Such clusters help to identify two subpopulations of either young or old patients whose etiologies are thought to be different. A large sample drawn from registry data from the National Cancer Institute's Surveillance, Epidemiology, and End Results program from 1990 to 2009 was analyzed using a two-component Gaussian mixture model. Evidence of a steady delay of age at diagnosis and an increasing proportion of young patients being diagnosed during the 20-year period was found. Histopathologic effects indicate that duct and lobular carcinomas differ significantly in regard to subpopulation membership, which confirms that they represent different etiologies. While the presence of estrogen receptor status in the model overlaps the effects of other important variables it is highly correlated with, it is found that the grade, extension and size of the tumor along with lymph node involvement status, race and marital status are important predictors of age at diagnosis. The results highlight the significant impacts that such features can have on breast cancer control efforts, and point to the importance of ensuring that medical decision making should use them along with an indicator of the age subpopulation a patient may belong to.
\end{abstract}

Keywords: Bimodality; Breast cancer etiology; Clustering; Histopathology; SEER; Split-population model

\section{Introduction}

Female breast cancer is a complex disease with different clinicopathologic features. Over the past three decades in the US, diagnostic methods have changed and female breast cancer etiology has evolved due to change in lifestyles and frequent screening. One of the primary challenges of breast cancer epidemiology is to identify risk factors such as age at onset which may provide clues for understanding the etiologic mechanisms of the disease. The ability to discriminate etiologically different subgroups in the population may eventually facilitate a number of research and clinical issues, including prevention and control strategies.

\footnotetext{
*Correspondence: ge@xanum.uam.mx Departement of Mathematics, Universidad Autónoma Metropolitana Iztapalapa, AT-351 UAM-I Av. San Rafael Atlixco No. 186 Col. Vicentina, Mexico City DF 09340, Mexico
}

It has been documented that early-onset female breast cancer differs from female breast cancer diagnosed at an older age in several ways. Firstly, female breast cancer diagnosed at a young age tends to have worse prognosis, namely fast-growing, high grade and hormone receptornegative tumors (Anders et al. 2008; Fei et al. 2013; Partridge et al. 2010), and as a consequence a higher cause-specific mortality (Aebi et al. 2000; Albain et al. 1994; Anders et al. 2008; de la Rochefordiere et al. 1993; El Saghir et al. 2006; Holli and Isola 1997; Kollias et al. 1997).

Secondly, age-specific breast cancer incidence patterns differ between young and old women which usually show an inflection point around menopause (Adami et al. 1986; El Saghir et al. 2006; Nixon et al. 1994) where women have been reported to have a reduced risk of breast cancer (Fei et al. 2013).

Although previous studies have found the existence of two main breast cancer subpopulations according to

\section{Springer}

(c) 2014 Escarela et al.; licensee Springer. This is an Open Access article distributed under the terms of the Creative Commons Attribution License (http://creativecommons.org/licenses/by/4.0), which permits unrestricted use, distribution, and reproduction in any medium, provided the original work is properly credited. 
age at onset, with the split point located around the menopause threshold (Anderson et al. 2002; Anderson and Matsuno 2006a; Anderson et al. 2006b; Tarone and Chu 2002; Yasui and Potter 1999), the etiology of these two groups is insufficiently understood and the question whether these subpopulations represent two different entities continues to be an open question; in addition, since it has been reported that changes in lifestyles over time have had influence in the occurrence of menarche, reproductive life and menopause in the US (Nichols et al. 2006), detecting and accounting for time-varying effects of age-at-diagnosis can provide insights on whether the characteristics at onset of the disease have also changed over time.

In this paper, the effects that temporal trend, clinicopathologic and sociodemographic variables have had on the distribution of age at diagnosis in a period of twenty years in the US is elucidated in order to understand the etiology of these two cancer subpopulations. A clustering method for identifying the two groups according to age at diagnosis in the presence of sociodemographic and clinicopathologic variables is implemented.

\section{Materials and methods}

\subsection{Data source}

Surveillance, Epidemiology, and End Results (SEER) Program data of newly diagnosed cases of female breast cancer from the years 1990 to 2009 were analyzed. SEER is a high-quality, population-based incidence data covering up to $26 \%$ of the US population. During the period of study, $N=446,726$ diagnosed cases of female breast cancer were registered in the nine SEER registries considered here, which include Atlanta, Connecticut, Detroit, Hawaii, Iowa, New Mexico, San Francisco-Oakland, Seattle-Puget Sound and Utah. Figure 1 depicts the histogram and kernel density curve of age-at-diagnosis for each year. Two key features are easily identified: a bimodal pattern and a notable change of both the shape and the location of the peaks. It can be noticed that, while the disease was more frequent in patients older than the inflection point during the first half of the 90's, the diagnosis became more common amongst younger women in the following years.

The variables that were considered as predictors of ageat-diagnosis were trend, which was computed as the orthogonal polynomial base of first degree for year + (month number -1$) / 12$, and the factors that are defined in uppercase as follows: SITE, the histopathologic subtype: 1) duct carcinomas, obtained from codes $8500-8508$ and $8521-8523(80 \%), 2)$ lobular carcinomas, obtained from codes 8520 and 8524 (9\%), and 3) Other (11\%); $\mathrm{ER}$, the estrogen receptor status: 1) positive (60\%), and 2 ) negative or borderline (17\%), with $23 \%$ missing; GRADE,

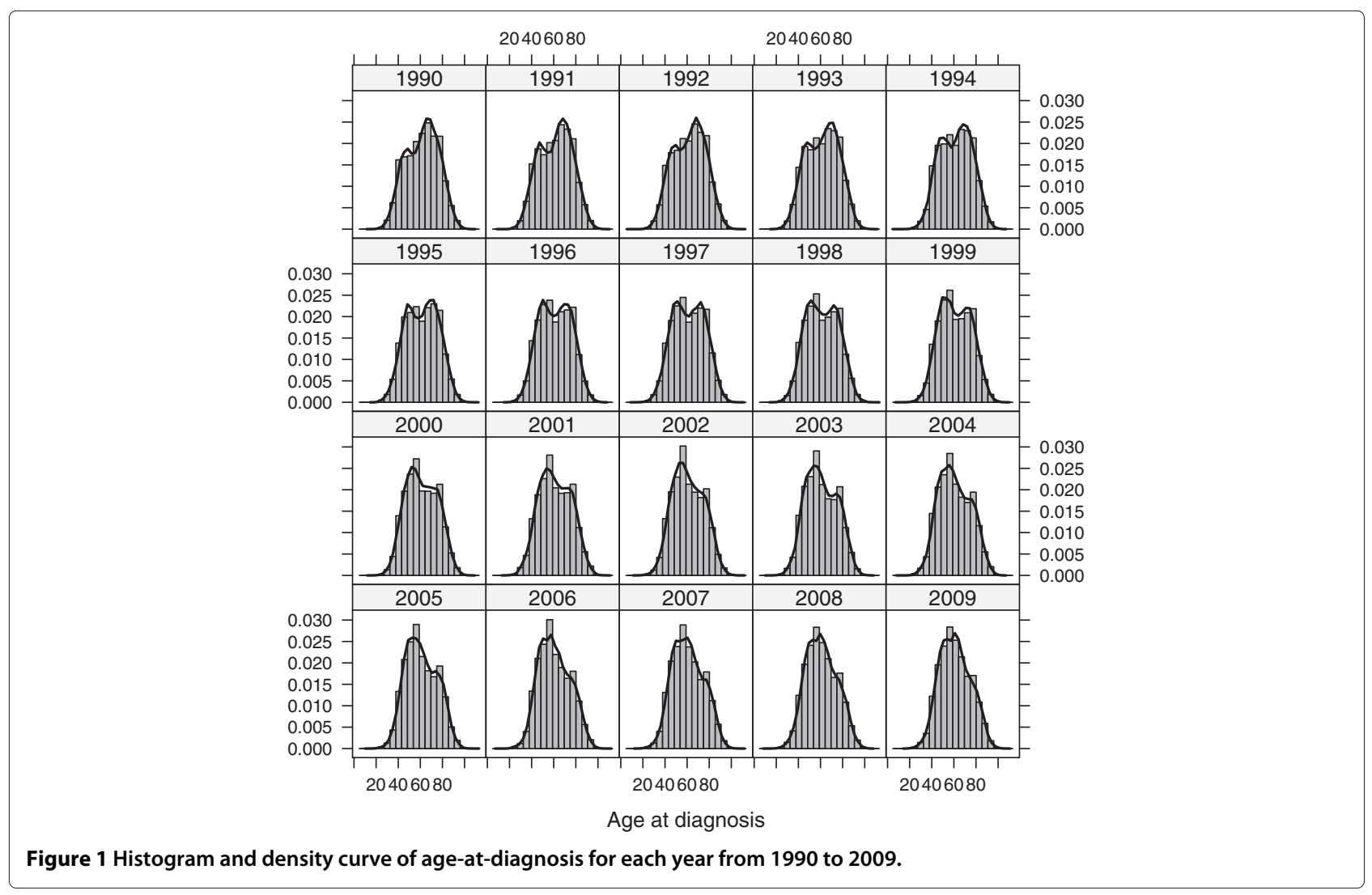


tumor grade: 1) well differentiated (16\%), 2) moderately differentiated (33\%), and 3) poorly differentiated, undifferentiated or anaplastic (31\%), with $20 \%$ missing; EXTENSION: 1) in situ or without underlying tumor or no evidence of it (18\%), 2) confined to breast tissue and fat including nipple and/or areola (72\%), and 3) Invasive components and further extension (8\%), with $2 \%$ missing; LYMPH: 1) no lymph node involvement (70\%), and 2) lymph node involvement (24\%), with $6 \%$ missing; SIZE, tumor size: 1) less than $2 \mathrm{~cm}(70 \%)$ and 2$) 2 \mathrm{~cm}$ or more (25\%), with 5\% missing; LATERALITY: 1) Right (49\%), and 2) left (51\%); RACE: 1) White (84\%), 2) Black (9\%) and 3) Asian or Pacific Islander (7\%); and MARRIED, marital status at diagnosis: 1) Single (never married, $12 \%$ ), and 2) Married (including common law), separated, divorced, widowed, unmarried or domestic partner (84\%), with $4 \%$ missing. Only one-sided laterality cases were considered in the dataset since cases with breast cancer in both sides, which were rare (less than .5\%), may have a different histopathology in each side. American Indians, Alaska natives and other unspecified races were not included in the study either (less than .1\%). Time-dependent variables were created by forming interaction terms trend by factor.

\subsection{Statistical methods}

The random variable $Y$ is defined as age at diagnosis and the model-based clustering technique employed here consists of a two-component mixture model to estimate both the underlying component distributions and the memberships of the two unlabelled groups. Specifically, the cumulative distribution function (c.d.f.) of the mixture model is defined by a weighted sum of two Gaussian component c.d.f's as follows:

$$
G(y)=(1-\pi) \Phi\left(\frac{y-\mu_{1}}{\sigma_{1}}\right)+\pi \Phi\left(\frac{y-\mu_{2}}{\sigma_{2}}\right)
$$

where $y$ takes all real values, $\Phi$ represents the c.d.f. of the standard normal, and the unknown mixture proportion $\pi$ $(0 \leq \pi \leq 1)$ along with the two within-cluster means $\mu_{1}$ and $\mu_{2}$, and the two within-cluster variances $\sigma_{1}^{2}$ and $\sigma_{2}^{2}$ are to be estimated; here the clusters indexed as 1 and 2 will be referred to as the young cluster and the old cluster, respectively. In order to include auxiliary variables in the split-population model in equation 1, the two withincluster means and variances are specified as functions of covariates as

$$
\mu_{k}(\mathbf{x})=\mathbf{x}^{T} \boldsymbol{\beta}_{k} \quad \text { and } \quad \sigma_{k}^{2}(\mathbf{x})=\exp \left(\mathbf{x}^{T} \boldsymbol{\gamma}_{k}\right)
$$

where $\mathbf{x}$ is the vector of explanatory variables which includes the intercept, $\boldsymbol{\beta}_{k}$ and $\boldsymbol{\gamma}_{k}$ are vectors of coef- ficients and $k=1,2$. In a similar way, the mixture proportion is specified by a logit function as follows

$$
\pi(\mathbf{x})=\frac{1}{1+\exp \left(\mathbf{x}^{T} \delta\right)},
$$

where $\delta$ is the vector of coefficients. A similar formulation has been proposed in Villani et al. 2009. Finite mixture models have been extensively discussed in Everitt and Hand 1981 and McLachlan and Basford 1988.

The type of estimators used in this study are obtained with maximum likelihood; here, the log-likelihood of the data $\left\{y_{1}, \ldots, y_{n}\right\}$ is $l(\boldsymbol{\theta})=\sum_{i=1}^{n} \log g\left(y_{i} ; \boldsymbol{\theta}\right)$, where $g(y ; \boldsymbol{\theta})$ is the density function corresponding to the c.d.f. of the mixture model in equation 1 and $\boldsymbol{\theta}$ is the vector that contains all unknown parameters. The log-likelihood can be maximized using general purpose optimizers to find the maximum likelihood estimators and the standard errors. In this study, the function $\mathrm{nlm}$ of the $\mathrm{R}$ language was used to optimize $l(\boldsymbol{\theta})$. The likelihood surface in the analysis presented here was well behaved and the optimizing procedure always led to the same solution for different starting values. The assumptions made about the mixture model may be checked by calculating the conditional randomized quantile residuals proposed by Dunn and Smyth (1996), which are defined by $r_{i}=\Phi^{-1}\left[\hat{G}\left(y_{i} ; \mathbf{x}_{i}\right)\right]$, where $\hat{G}\left(y_{i} ; \mathbf{x}_{i}\right)$ is the fitted cumulative distribution function and $i=1, \ldots, n$. Since such residuals are exactly normal under the assumed model, some simple plots for checking that they are observed values of independent and standard normal random variates should indicate the quality of the fit.

The coding used to create the dummy variables corresponding to the levels of the factors was treatment contrasts (Chambers and Hastie 1992), which sets the coefficients of the baseline level in each categorical variable equal to 0; here, the baseline level is taken as the first category of the corresponding factor as described above. Since around $40 \%$ of the cases in the entire dataset contain at least a missing predictor, the principled method of dealing with the missing data employed here was multiple imputation. The inference procedure consisted of the generation of multiple stochastically "completed" datasets using the mice package in the $\mathrm{R}$ statistical language (van Buuren and Groothuis-Oudshoorn 2011), which uses a chained equations algorithm, then each completed data set was analyzed using the model for complete data, and finally the results were combined using Rubin's rules (Rubin 1987).

Since the implementation of the chained equations algorithm is computationally expensive for large datasets, a random sample of size $n=20,000$ was obtained, from which forty imputations were generated. The resulting five-number-summary of trend in the sample analyzed 
here was: minimum $=-0.0132$, 25 th percentile $=-$ 0.0058 , median $=0.0003,75$ th percentile $=0.0061$ and maximum $=0.0116$. It was assumed that the missing data mechanism is missing at random (MAR) (Little and Rubin 2002), which specifies that the probability that a data value is missing depends on values of variables that were actually measured. Including as many variables in the imputation model as possible yields multiple imputations that tend to minimize bias and make the MAR assumption more plausible, which reduces the need to make special adjustments for more complex missing data mechanisms (Schafer 1997).

The problem of variable selection in the mixture model was addressed with the "impute, then select" strategy, which involves initially performing multiple imputation and subsequently applying Bayesian variable selection to each of the enhanced datasets (Yang et al. 2005). The variables included in the final model appear in at least 50 per cent of the selected models obtained in the imputed datasets (Wood et al. 2008). To determine the most appropriate covariates to be included in the model of each imputed dataset, the Bayesian information criterion (BIC) (Schwarz 1978) was adopted as the main model choice criterion. If $n_{p}$ denotes the number of parameters in the model and $n$ the number of individuals in the dataset, the BIC criterion is to choose the model for which $-2 \hat{l}+$ $n_{p} \log (n)$ is the smallest; here, $\hat{l}$ is the maximized loglikelihood function. Backward elimination was employed to arrive at the best fitting model in each imputed dataset. Both the variable selection process and the combined results were based on the forty enhanced datasets.

\section{Results}

When obtaining the most parsimonious model, it was found that, in the presence of trend and all factors, no time-varying variable had any impact on age-at-diagnosis, which suggests that the features at onset of the disease have not substantially changed during the twenty-year period studied here. Also, laterality was eliminated from the best fitting model, which indicates that it is not associated with the age at onset of breast cancer, confirming results from other studies (Weiss et al. 1996).

Although ER was selected in the most parsimonious model and it showed very significant effects when it was the only covariate included in the mixture model, it was noticed that, while its individual effects were non significant in the most parsimonious model, its presence made more significant most of the remaining variables. This suggests that the simultaneous inclusion of ER with other important auxiliary variables causes a problem of multicollinearity, in the sense that ER is correlated with some of the auxiliary variables. As a matter of fact, the association between ER and other important breast cancer histologic variables has been documented to be high, particularly with the grade of the tumor (Fisher et al. 1981; Lal et al. 2005). In this study, for samples of size 1000 of each enhanced dataset, the null hypothesis of independence between ER and GRADE was rejected for each sample at a significance level of 0.001 when performing the chi-squared contingency table test; in addition, the null hypothesis of ER being independent of SITE and SIZE was rejected for each sample at the significance level of 0.01 . Since the presence of ER clearly overlaps the effects of other important variables, which causes overestimation and thereby an artificial inflation, it was decided that ER would not be included in the analysis.

Table 1 shows parameter estimates for the best fitting two-component Gaussian mixture model. The most significant coefficients correspond to trend, whose positive signs in the beta coefficients indicate that the two medians have moved to the right with time, which confirms that age at diagnosis has had a delaying effect with respect to time; in addition, the opposite signs of the gamma coefficients suggests that the young cluster has become more heterogeneous whilst the old cluster has become more homogeneous, and the negative delta coefficient implies that the age at onset has become more common for younger patients. When it comes to comparing the histopathologic subtypes, the coefficients show marked discrepancies between the duct and the lobular carcinomas. Broadly, lobular carcinomas tended to be diagnosed in women at earlier ages than women with duct carcinomas, particularly for the old cluster, and duct carcinomas have been more prevalent in older patients. There are no significant differences between duct carcinomas and other subtypes of carcinomas when categorized as a whole.

Coefficients corresponding to clinicopathologic variables indicate that high-grade cancers have been more frequent in the young cluster and invasive components and further extension tumors have been diagnosed at later ages than other levels of extension in both clusters, particularly for the old cluster. Also, contrary to other findings where no differences have been identified for lymph node status and tumor size amongst groups of age (Kollias et al. 1997), the results here indicate that, while lymph node involvement was more likely to be present in younger patients than those without it, bigger tumors were associated with older women in the old cluster, which was not the case amongst women in the young cluster.

In regard to sociodemographic characteristics, the coefficients suggest that Blacks and Asians or Pacific Islanders have tended to be more frequently diagnosed in the young cluster than their White counterparts; in addition, patients with breast cancer who were married at the time of diagnosis were more frequent in the older cluster, and women in the young cluster tended to be diagnosed at later ages than women with no partners, which may 
Table 1 Parameter estimates (standard errors in brackets) of the best fitting two-component Gaussian mixture model for the regression of age-at-diagnosis of female breast cancer among US women diagnosed from 1990 to 2009 based on 40 imputations of a sample of size $n=20,000$

\begin{tabular}{|c|c|c|c|c|c|}
\hline Covariate & $\beta_{1}$ & $\beta_{2}$ & $\gamma_{1}$ & $\gamma_{2}$ & $\delta$ \\
\hline Intercept & $50.29 * * *(1.111)$ & $71.91^{* * *}(2.027)$ & $4.039^{* * *}(0.160)$ & $4.172^{* * *}(0.237)$ & $-0.793 *(0.337)$ \\
\hline trend & $347.3^{* * *}(37.97)$ & $363.1^{* * *}(50.02)$ & $25.92^{* * *}(4.748)$ & $-20.28^{* * *}(6.118)$ & $-73.57^{* * *}(8.561)$ \\
\hline $\mathrm{SITE}_{2}$ & $-1.901^{* *}(0.732)$ & $-4.311^{* * *}(1.149)$ & $-0.387^{* *}(0.125)$ & $0.359^{* *}(0.119)$ & $0.713^{* *}(0.234)$ \\
\hline $\mathrm{SITE}_{3}$ & $0.594(0.820)$ & $0.904(0.812)$ & $0.099(0.100)$ & $0.181(0.100)$ & $0.292(0.156)$ \\
\hline $\mathrm{GRADE}_{2}$ & $-0.007(0.692)$ & $0.351(0.725)$ & $0.211 *(0.096)$ & $-0.033(0.094)$ & $-0.257(0.143)$ \\
\hline $\mathrm{GRADE}_{3}$ & $-1.368(0.744)$ & $-0.172(0.870)$ & $0.287^{* *}(0.105)$ & $0.009(0.110)$ & $-0.698^{* * *}(0.160)$ \\
\hline EXTENSION 2 & $0.863(0.890)$ & $2.686(1.693)$ & $0.245^{*}(0.121)$ & $0.040(0.199)$ & $0.314(0.293)$ \\
\hline EXTENSION $_{3}$ & $4.089^{* * *}(1.189)$ & $6.573^{* * *}(1.781)$ & $0.355^{*}(0.159)$ & $-0.147(0.211)$ & $0.455(0.311)$ \\
\hline $\mathrm{LYMPH}_{2}$ & $-2.380^{* * *}(0.571)$ & $-2.658^{* * *}(0.704)$ & $-0.100(0.073)$ & $0.113(0.090)$ & $-0.165(0.122)$ \\
\hline $\mathrm{SIZE}_{2}$ & $-0.540(0.560)$ & $2.060^{* *}(0.693)$ & $0.125(0.070)$ & $0.088(0.085)$ & $-0.088(0.120)$ \\
\hline $\mathrm{RACE}_{2}$ & $-0.562(0.738)$ & $-1.174(1.221)$ & $0.057(0.093)$ & $-0.007(0.147)$ & $-0.433^{*}(0.194)$ \\
\hline $\mathrm{RACE}_{3}$ & $-0.708(0.863)$ & $-1.428(1.151)$ & 0.109 (0.108) & $-0.133(0.149)$ & $-0.462^{*}(0.211)$ \\
\hline $\mathrm{MARRIED}_{2}$ & $1.961^{* * *}(0.567)$ & $-0.696(0.979)$ & $-0.185^{*}(0.075)$ & $0.005(0.125)$ & $0.845^{* * *}(0.143)$ \\
\hline
\end{tabular}

${ }^{*} p$-value $<0.05,{ }^{* *} p$ - value $<0.01,{ }^{* * *} p$ - value $<0.001$

support the common belief that having a partner leads to a healthier lifestyle regarding substance use, physical activity and social support, delaying the onset of the disease amongst those susceptible (Bailey 2009).

A review of the randomized quantile residual plots for each enhanced dataset, which consisted of density, quantile-quantile, autoregressive and partial autoregressive plots, provided assurance that there is fairly normal distribution and independence of the residuals, which indicates that the model assumptions were met.

\section{Discussion}

This study has characterized the distribution of age at diagnosis in the North American population and examined factors that are related to this distribution. The results are based on a large sample from a populationbased data-base collected in the US between 1990 and 2009. The sample analyzed here combines histopathology, and clinicopathologic, sociodemographic and trend variables. The comprehensive statistical analysis of the data along with the corresponding residual analysis confirms that such distribution is a mixture of two different populations. A strength of the present analysis is the number of patients from which trend, clinicopathologic and sociodemographic data are available; however, it is limited by the standardization of the clinicopathologic variables across time, the usual concerns related to registry data. This analysis hopefully provides a clearer understanding into the complexity driving age at onset of female breast cancer.

The most remarkable finding of the analysis was that temporal trend is the most important variable in determining the shapes, modes and probability of membership of the two populations over the 20-year follow up, which may suggest that, changes in reproductive and lifestyle factors, such as childbearing, eating habits (Tretli and Gaard 1996) and exposure to both exogenous and endogenous estrogens (Chen 2008), along with more frequent and different types of screening have had an important impact on the age at diagnosis. The main characteristic of trend was a steadily increasing age at diagnosis and a decreasing proportion of older women being diagnosed. Since no interaction trend by factor showed significant effects, it can be inferred that both clinicopathologic and sociodemographic variables have not been influenced enough by the embedded features that such trend may carry on their joint association with age at diagnosis. The results support findings from other research that duct and lobular carcinomas, the most common breast carcinomas, represent two different etiologies. As a matter of fact, other studies suggest that such carcinomas may be biologically different malignancies and have different risk factors (Pike et al. 1993).

There is much interest in estimating the inflection point between the two component densities in the mixture model in order to define a split-point that allows for the determination of the memberships in the two cancer populations in age-specific breast cancer incidence. In other studies, such split-point has been claimed to be around menopause (e.g. Anderson et al. 2006b; Yasui and Potter 1999). In this study, the p.d.f. of the mixture model has a complex interplay between the component means and variances and the mixture proportions in which the resulting p.d.f. is not necessarily bimodal, which makes difficult to analytically estimate the split-point for the 
different factor combinations and trend. Considering that menopause has also been delayed in North America due to the aforementioned lifestyle factors (Nichols et al. 2006) and that recent research supports a low breast cancer risk around this part of a woman's life (Fei et al. 2013; Huang et al. 2011), it seems reasonable to speculate that the splitpoint is menopause. Nevertheless, the results obtained here indicate that the coefficients of sociodemographic and clinicopathologic variables in $\delta$ are important predictors for determining $1-\pi$, the probability of membership in the young cluster, and $\pi$, the probability of membership in the old cluster. Such probabilities can be easily computed using the logit function in equation 3 and then be employed as a criterion in order to decide to which cluster a patient may belong, which could potentially improve the decision-making in patient management and survival.

The problem of multicollinearity in the model helped to confirm that ER status has a high degree of association with tumor grade, the histopathologic subtype and tumor size. Despite the recognised role of ER as a proxy for prognosis in medical decision making, it has been documented that hormone receptors such as ER are relatively weak predictors for determining outcome in patients diagnosed with breast cancer and are of limited clinical value in lymph node-negative cases (Mirza et al. 2002), which confirms that treatment guidelines based on ER alone are ill-defined. The results obtained here suggest that age at diagnosis, histopathologic subtype, tumor grade, tumor extension, lymph node involvement, tumor size, race and marital status at diagnosis along with other well established prognostic factors should jointly be used in order to build a conceptual framework for a more rational therapeutic approach to breast cancer. Given these considerations, the need for further research in this area is obvious.

\section{Competing interests}

The authors declare that they have no competing interests.

\section{Authors' contributions}

GE and GNA both participated in the conception and design of this study. LCPR was critical to the acquisition and analysis of the data. All authors have reviewed and given final approval for this version of the paper to be published.

\section{Acknowledgments}

The authors are grateful to the Surveillance, Epidemiology, and End Results program of the National Cancer Institute for providing access to the database. The authors would like to thank CONACYT, the Mexican Research Council, for the funding of their research.

Received: 9 October 2014 Accepted: 10 October 2014 Published: 23 October 2014

\section{References}

Adami HO, Malker B, Holmberg L, Persson I, Stone B (1986) The relation between survival and age at diagnosis in breast cancer. $N$ Engl $J$ Med 315:559-563

Aebi S, Gelber S, Castiglione-Gertsch M, Gelber RD, Collins J, Thürlimann B, Rudenstam CM, Lindtner J, Crivellari D, Cortes-Funes H, Simoncini E, Werner ID, Coates AS, Goldhirsch A (2000) Is chemotherapy alone adequate for young women with oestrogen receptor-positive breast cancer Lancet 355:1869-1874

Albain KS, Allred DC, Clark GM (1994) Breast cancer outcome and predictors of outcome: Are there age differentials I Natl Cancer Inst Monogr 16: $35-42$

Anders CK, Hsu DS, Broadwater G, Acharya CR, Foekens JA, Zhang Y, Wang Y, Marcom PK, Marks JR, Febbo PG, Nevins JR, Potti A, Blackwell KL (2008) Young age at diagnosis correlates with worse prognosis and defines a subset of breast cancers with shared patterns of gene expression. J Clin Oncol 26(20):3324-30. doi:10.1200/JCO.2007.14.2471

Anderson WF, Chatterjee N, Ershler WB, Brawley OW (2002) Estrogen receptor breast cancer phenotypes in the Surveillance, Epidemiology, and End Results database. Breast Cancer Res Treat 76:27-36

Anderson WF, Matsuno RK (2006a) Breast cancer heterogeneity: a mixture of at least two main types. J Natl Cancer Inst 98:948-51

Anderson WF, Pfeiffer RM, Dores GM, Sherman ME (2006b) Comparison of age distribution patterns for different histopathologic types of breast carcinoma. Cancer Epidemiol Biomarkers Prev 15:1899-1905

Bailey J (2009) Effects of marital status on cancer incidence and survival rates. Am Fam Physician 80:1052

Chambers JM, Hastie TJ (1992) Statistical models. In: Chambers JM, Hastie TJ (eds) Statistical models in S. Wadsworth \& Brooks/Cole, Pacific Grove CA. pp 13-44

Chen WY (2008) Exogenous and endogenous hormones and breast cancer. Best Pract Res Clin Endocrinol Metab 22(4):573-85

de la Rochefordiere A, Asselain B, Campana F, Scholl SM, Fenton J, Vilcoq JR, Durand JC, Pouillart P, Magdelenat H, Fourquet A (1993) Age as prognostic factor in premenopausal breast carcinoma. Lancet 341:1039-1043

Dunn PK, Smyth GK (1996) Randomized quantile residuals. J Comput Graph Stat 5:236-244

El Saghir NS, Seoud M, Khali MK, Charafeddine M, Salem ZK, Geara FB, Shamseddine Al (2006) Effects of young age at presentation on survival in breast cancer. BMC Cancer 6:194

Everitt BS, Hand DJ (1981) Finite mixture distributions. Springer, London

Fei C, DeRoo LA, Sandler DP, Weinberg CR (2013) Menopausal symptoms and the risk of young-onset breast cancer. Eur J Cancer 49(4):798-804. doi:10.1016/j.ejca.2012.08.030

Fisher ER, Osborne CK, McGuire WL, Redmond C, Knight WA 3rd, Fisher B, Bannayan G, Walder A, Gregory EJ, Jacobsen A, Queen DM, Bennett DE, Ford HC (1981) Correlation of primary breast cancer histopathology and estrogen receptor content. Breast Cancer Res Treat 1(1):37-41

Holli K, Isola J (1997) Effect of age on the survival of breast cancer patients. Eur J Cancer 33:425-428

Huang Y, Malone KE, Cushing-Haugen KL, Daling JR, Li Cl (2011) Relationship between menopausal symptoms and risk of postmenopausal breast cancer. Cancer Epidemiol Biomarkers Prev 20(2):379-88

Kollias J, Elston CW, Ellis IO, Robertson JF, Blamey RW (1997) Early onset breast cancer: histopathologic and prognostic considerations. Br J Cancer 75:1318-1323

Lal P, Tan LK, Chen B (2005) Correlation of HER-2 status with estrogen and progesterone receptors and histologic features in 3,655 invasive breast carcinomas. Am J Clin Pathol 123(4):541-6

Little RJA, Rubin DB (2002) Statistical analysis with missing data. 2nd edn. Wiley, Hoboken NJ

McLachlan GJ, Basford KE (1988) Mixture models: inference and applications to clustering. Marcel Dekker, New York

Mirza AN, Mirza NQ, Vlastos G, Singletary SE (2002) Prognostic factors in node-negative breast cancer: a review of studies with sample size more than 200 and follow-up more than 5 years. Ann Surg 235(1):10-26

Nichols HB, Trentham-Dietz A, Hampton JM, Titus-Ernstoff L, Egan KM, Willett WC, Newcomb PA (2006) From Menarche to Menopause: trends among US women born from 1912 to 1969. Am J Epidemiol 164(10): 1003-1011

Nixon AJ, Neuburg D, Hayes DF, Gelman R, Connolly JL, Schnitt S, Abner A, Recht A, Vicini F, Harris JR (1994) Relationship of patient age to pathologic features of the tumor and prognosis for patients with stage I and II breast cancer. J Clin Oncol 12:888-894

Partridge AH, Goldhirsch A, Gelber S, Gelber RD (2010) Chapter 92: Breast Cancer in Younger Women. In: Harris JR, Lippman ME, Morrow M, Osborne CK (eds) Diseases of the Breast, 4th edn. Lippincott Williams \& Wilkins, Philadelphia Pa 
Pike MC, Spicer DV, Dahmoush L, Press MF (1993) Estrogens, progestogens, normal breast cell proliferation, and breast cancer risk. Epidemiol Rev 15(1):17-35

Rubin DB (1987) Multiple imputation for nonresponse in surveys. John Wiley and Sons, New York

Schafer JL (1997) Analysis of incomplete multivariate data. Chapman and Hall, New York

Schwarz GE (1978) Estimating the dimension of a model. Annals Stat 6(2):461-464. doi:10.1214/aos/1176344136

Tarone RE, Chu KC (2002) The greater impact of menopause on ER- than ER+ breast cancer incidence: a possible explanation (United States). Cancer Causes Control 13:7-14

Tretli S, Gaard M (1996) Lifestyle changes during adolescence and risk of breast cancer: an ecologic study of the effect of World War II in Norway. Cancer Causes Control 7(5):507-12

van Buuren S, Groothuis-Oudshoorn K (2011) mice: Multivariate Imputation by Chained Equations in R. J Stat Softw 45(3):1-67

Villani M, Kohn R, Giordani P (2009) Regression density estimation using smooth adaptive Gaussian mixtures. J Econometrics 153:155-173

Weiss HA, Devesa SS, Brinton LA (1996) Laterality of breast cancer in the United States. Cancer Causes Control 7(5):539-43

Wood AM, White IR, Royston P (2008) How should variable selection be performed with multiply imputed data Stat Med 27(17):3227-46

Yang X, Belin TR, Boscardin WJ (2005) Imputation and variable selection in linear regression models with missing covariates. Biometrics 61(2):498-506

Yasui Y, Potter JD (1999) The shape of age-incidence curves of female breast cancer by hormone-receptor status. Cancer Causes Control 10:431-7

doi:10.1186/2193-1801-3-626

Cite this article as: Escarela et al.: Temporal trend, clinicopathologic and sociodemographic characterization of age at diagnosis of breast cancer among US women diagnosed from 1990 to 2009. SpringerPlus 2014 3:626.

\section{Submit your manuscript to a SpringerOpen ${ }^{\odot}$ journal and benefit from:}

- Convenient online submission

- Rigorous peer review

- Immediate publication on acceptance

- Open access: articles freely available online

- High visibility within the field

- Retaining the copyright to your article

Submit your next manuscript at $\boldsymbol{\triangleright}$ springeropen.com 